\title{
Lobectomia com Ressecção em Bloco de Parede Torácica por Vídeotoracoscopia para Tratamento do Câncer de Pulmão
}

\author{
Lobectomy with Resection in Block of Thoracic Wall by Video- \\ Assisted Thoracoscopy for Treatment of Lung Cancer
}

Ricardo Oliveira ${ }^{1}$

${ }^{1}$ Serviço de Cirurgia Torácica, Hospital Santa Izabel; Salvador, Bahia, Brasil

\begin{abstract}
Correspondence addresses:
Dr. Ricardo Oliveira ricardooliveira@cirtoraxbahia. com.br
\end{abstract}

Received: April 13, 2019

Revised: May 31, 2019

Accepted: June 4, 2019

Published: June 28, 2019

Data Availability Statement: All relevant data are within the paper and its Supporting Information files.

Funding: This work was the result of author's initiative. There was no support of research or publication funds.

Competing interests: The author has declared that no conflit of interests exists.

Copyright

(C) 2019 by Santa Casa de Misericórdia da Bahia.

All rights reserved.

ISSN: 2526-5563
A $8^{\mathrm{a}}$ edição do sistema de estadiamento TNM classificou a neoplasia primária de pulmão com invasão da pleura parietal e parede torácica como T3, com sobrevida em 5 anos de $40 \%-50 \%$ na ausência de metástase para linfonodos. $O$ tratamento padrão preconizado é a ressecção da parede torácica como parte do tratamento cirúrgico em conjunto com a lobectomia. Entretanto, mesmo escassa a literatura, benefícios da cirurgia minimamente invasiva por vídeotoracoscopia (VATS) tem sido reportada, sendo a lobectomia por este método a escolha para as neoplasias de pulmão de estágio inicial. $O$ aperfeiçoamento das técnicas de vídeotoracoscopia e o crescimento da experiência dos serviços com ressecções pulmonares complexas por VATS vêm aumentando a aceitação desta abordagem como método de escolha em casos específicos.

Palvras-chave: Câncer de Pulmão; Lobectomia; Vídeotoracoscopia; Estadiamento.

The $8^{\text {th }}$ edition of the TNM staging system classified primary lung neoplasm with invasion of the parietal pleura and chest wall as T3, with 5-year survival of $40 \%$ $-50 \%$ in the absence of lymph node metastasis. The recommended standard treatment is resection of the chest wall as part of the surgical treatment in conjunction with the lobectomy. However, notwithstanding, sparse literature have been reporting the benefits of minimally invasive videothoracoscopy surgery (VATS). Nowadays, lobectomy though VATS is the method of choice for surgical treatment in early stage lung cancer. The improvement of the techniques of videothoracoscopy and the growth of the experience of the services with complex lung resections by VATS have increased the acceptance of this approach in specific cases.

Keywords: Lung Cancer; Lobectomy; Videothoracoscopy; Staging.

\section{Introdução}

A incidência de neoplasia primária de pulmão com invasão da pleura parietal e parede torácica é de $5 \%-8 \%$ dos casos. ${ }^{1} \mathrm{Na} 8^{\text {a }}$ Edição do sistema de estadiamento TNM, esses tumores são classificados como $\mathrm{T}^{2} \mathrm{e}$, na ausência de metástase para linfonodos, a sobrevida em 5 anos é de $40 \%$ $50 \% .{ }^{3,4}$ A ressecção da parede torácica como parte do tratamento cirúrgico em conjunto com a lobectomia é o tratamento padrão preconizado. Historicamente, a ressecção cirúrgica por via aberta ou toracotomia foi a 
técnica mais utilizada no tratamento cirúrgico do câncer de pulmão. No entanto, o reconhecimento dos benefícios da cirurgia minimamente invasiva por vídeotoracoscopia (VATS) em termos de menor taxa de complicações e resultados oncológicos semelhantes vem modificando esse cenário. ${ }^{5}$ Atualmente, a lobectomia por VATS é o método de escolha para tratamento cirúrgico das neoplasias de pulmão de estágio inicial. ${ }^{6} \mathrm{Em}$ relação à doença mais avançada, como é o caso da invasão de parede torácica, a factibilidade de realizar ressecções mais complexas por VATS é questionada, principalmente devido ao risco de comprometimento de princípios oncológicos como a obtenção de margens cirúrgicas livres de neoplasia. Apesar desta desconfiança, algumas séries foram publicadas na literatura reportando a segurança e viabilidade da lobectomia com ressecção de parede torácica por VATS ${ }^{7-10}$ sob o racional de reduzir o trauma cirúrgico e oferecer os benefícios da técnica minimamente invasiva e com preservação dos resultados oncológicos.

\section{Quadro Clínico}

O sintoma típico dos pacientes portadores de neoplasia pulmonar cominvasão de parede torácica é dor de forte intensidade e de difícil controle. Entretanto, a ausência da dor não é critério de exclusão para invasão da parede, ${ }^{11}$ assim como sua presença não é patognomônica. ${ }^{12}$ Nos casos em que não há invasão grosseira nos exames de imagem, a combinação entre dor localizada e o íntimo contato do tumor com a parede torácica reforça bastante a suspeita e, muitas vezes, a confirmação da invasão é feita no intraoperatório.

\section{Estadiamento}

$\mathrm{O}$ estadiamento adequado do câncer de pulmão é crucial para definição do tratamento. Os principais exames de imagem utilizados com esse objetivo são: tomografia computadorizada do tórax e abdome superior; tomografia com emissão de pósitrons (PET-CT); tomografia ou ressonância do sistema nervoso central. ${ }^{13,14}$ A definição do status linfonodal do mediastino tem valor prognóstico e de definição de conduta significativo e todos pacientes com neoplasia de pulmão e suspeita de invasão de parede devem prosseguir com biópsia de linfonodos mediastinais através da ecobroncoscopia (EBUS) ou mediastinoscopia. ${ }^{5}$ Neste grupo de pacientes, a presença de metástase para linfonodos mediastinais (T3N2) determina uma redução importante na sobrevida global em 5 anos quando comparado à população sem disseminação para o mediastino (T3N0), $18 \%$ e $60 \%$, respectivamente. ${ }^{15}$ A detecção préoperatória de doença $\mathrm{N} 2$ em pacientes com invasão de parede geralmente exclui a cirurgia do manejo terapêutico.

\section{Tratamento Cirúrgico}

Os principais fatores preditivos de sobrevida livre de doença e sobrevida global em pacientes com câncer de pulmão com invasão de parede torácica são ausência de metástase para linfonodos mediastinais e ressecção completa com margens livres de neoplasia. ${ }^{16}$ Portanto, a investigação pré-operatória do status linfonodal mediastinal deve ser exaustiva conforme descrito anteriormente. Em relação à ressecção cirúrgica, preferencialmente, oobjetivoérealizara ressecção pulmonar (lobectomia ou pneumonectomia) e da parede torácica (toracectomia) em bloco. Não há consenso em relação ao tamanho das margens cirúrgicas da toracectomia, porém preconiza-se que seja de pelo menos $1 \mathrm{~cm}$ em todas as direções ou de 1 costela intacta acima e abaixo do tumor e $3-4 \mathrm{~cm}$ de margem lateral. ${ }^{15}$

A cirurgia de ressecção pulmonar e parede torácica em bloco estão associadas à morbimortalidade maiores do que o esperado para uma lobectomia por VATS, alcançando taxas de $20 \%$ e $3,8 \%-7,0 \%$, respectivamente. ${ }^{17,18}$ As complicações pulmonares são as mais comuns. ${ }^{19}$

Racionalmente, uma estratégia para tentar reduzir complicações, seria adotar técnicas minimamente invasivas. Não há na literatura trabalhos que compararam toracotomia versus VATS no contexto de tumores T3N0 com invasão 
de parede, e a escolha da técnica depende da expertise do cirurgião em cirurgia minimamente invasiva e da aceitação ou não de que a VATS resulta em menor trauma cirúrgico, mesmo neste paciente que necessita de toracectomia.

A literatura é escassa sobre ressecções pulmonares e parede torácica em bloco por vídeotoracoscopia, com poucas publicações de séries pequenas e relatos de caso..$^{7-10}$

A cirurgia pode ser realizada com múltiplos portais ${ }^{20}$ ouuniportal einiciadapela toracectomia ${ }^{20}$ ou pela ressecção pulmonar, ${ }^{5}$ a depender da preferência do serviço. A secção dos músculos intercostais e dos arcos costais pode ser alcançada através de uma contra-incisão ${ }^{5}$ ou pela própria incisão de trabalho da VATS. ${ }^{20} \mathrm{Na}$ primeira situação, a vídeotoracoscopia auxilia a delimitar precisamente a extensão de parede torácica que precisa ser ressecada e a determinar o melhor local para confecção da contra-incisão na qual instrumentais cortantes de cirurgia aberta são utilizados para seccionar as costelas. Na segunda situação, os arcos costais são seccionados com auxílio de cortadores de costelas endoscópicos sem necessidade de contra-incisão.

No serviço de cirurgia torácica da Universidade Laval em Quebec-CAN, a ressecção pulmonar e parede torácica em bloco são realizadas por VATS uniportal, iniciando pela lobectomia, seguido pela delimitação da área a ser ressecada e, finalmente, realizando-se a toracectomia por uma contra-incisão.

A reconstrução do defeito da parede torácica após a ressecção nem sempre é necessária. Nos tumores de localização posterior, atrás da escápula e menores que $5 \mathrm{~cm}$, geralmente a reconstrução é dispensável. Quando indicada, a correção do defeito da parede torácica pode ser feita com material flexível tipo tela ${ }^{21,22}$ ou rígido tipo barras metálicas. ${ }^{23}$

\section{Terapias Adjuvante e Neoadjuvante}

O papel da terapia adjuvante é controversa em tumores T3N0. Em pacientes com tumores $>4 \mathrm{~cm}$, pode-se considerar quimioterapia adjuvante com possível ganho de sobrevida baseado em análise de subgrupo do estudo CALGB 9633. ${ }^{24}$ No caso de ressecção incompleta com margens comprometidas, ampliação cirúrgica das margens ou radioterapia pós-operatória podem ser utilizadas. ${ }^{25}$

Em relação à terapia neoadjuvante, a literatura é ainda mais limitada para suportar a indicação de quimioterapia com ou sem radioterapia préoperatória em tumores com invasão de parede torácica. No entanto, essa abordagem vem sendo estudada, mostrando-se possivelmente segura e com taxas de resposta patológica elevadas. ${ }^{26}$

\section{Conclusão}

O tratamento cirúrgico de tumores de pulmão com invasão da parede através de ressecção pulmonar e da parede torácica em bloco por vídeotoracoscopia é factível. Através da cirurgia minimamente invasiva, este grupo de pacientes pode se beneficiar de menos dor pósoperatória, menor tempo de internação e melhor recuperação. $\mathrm{O}$ aperfeiçoamento das técnicas de vídeotoracoscopia e o crescimento da experiência dos serviços com ressecções pulmonares complexas por VATS vêm aumentando a aceitação desta abordagem, traduzindo-se em um número maior de publicações. Estudos com maior tempo de seguimento e avaliando os resultados oncológicos são necessários para consolidação desta técnica.

\section{Referências}

1. Grillo HC. Technical consideration in stage III disease: pleural and chest wall involvement. In: Delarue $\mathrm{NC}$, Eschapasse $\mathrm{H}$, editors. International Trends in General Thoracic Surgery. New York, NY: Saunders. 1985;134-8.

2. Detterbeck FC, Boff DJ, Kim AW, et al. The eighth edition lung cancer stage classification. Chest 2017;151:193-203.

3. Downey RJ, Martini N, Rusch VW, et al. Extent of chest wall invasion and survival in patients with lung cancer. Ann Thorac Surg 1999;68:188-93. 
4. Matsuoka H, Nishio W, Okada M, et al. Resection of chest wall invasion in patients with non-small cell lung cancer. Eur J Cardiothorac Surg. 2004;26:1200-4.

5. Berry MF, Onaitis MW, Tong BC, et al. Feasibility of hybrid thoracoscopic lobectomy and en-bloc chest wall resection. Eur J Cardiothorac Surg. 2012;41:888-92.

6. Bendixen $\mathrm{M}$, Jorgensen $\mathrm{OD}$, Kronborg $\mathrm{C}$, et al. Postoperative pain and quality of life after lobectomy via video-assisted thoracoscopic surgery or anterolateral thoracotomy for early stage lung cancer: a randomized controlled trial. Lancet Oncol. 2016;17:836-44.

7. Rocco G, Fazioli F, Martucci N, et al. Video-assisted thoracic surgery rib resection and reconstruction with titanium plate. Ann Thorac Surg. 2011;92:744-5.

8. Nakagiri T, Akashi A, Shigemura N. Thoracoscopic rib resection using a Gigli saw. Ann Thorac Surg 2005;80:755-6.

9. Abicht TO, de Hoyos AL. Chest wall resection and reconstruction: a true thoracoscopic approach. Innovations (Phila) 2011;6:399-402.

10. Widmann MD, Caccavale RJ, Bocage JP, et al. Videoassisted thoracic surgery resection of chest wall en bloc for lung carcinoma. Ann Thorac Surg. 2000;70:213840.

11. Lanuti M. Surgical managementoflung cancerinvolving the chest wall. Thorac Surg Clin. 2017;27:195-9.

12. Stoelben E, Ludwig C. Chest wall resection for lung cancer: indications and techniques. Eur J Cardiothorac Surg. 2009;35:450-6.

13. NCCN. Clinical Practice Guidelines in Oncology: Non- Small Cell Lung Cancer. Version 3. 2018.

14. De Leyn P, Dooms C, Kuzdzal J, et al. Revised ESTS guidelines for preoperative mediastinal lymph node staging for non-small-cell lung cancer. Eur J Cardiothorac Surg. 2014;45:787-98.

15. Facciolo F, Cardillo G, Lopergolo M, et al. Chest wall invasion in non-small cell lung carcinoma: a rationale for en bloc resection. J Thorac Cardiovasc Surg. 2001;121:649-56.

16. Voltolini L, Rapicetta C, Luzzi L, et al. Lung cancer with chest wall involvement: Predictive factors of longterm survival after surgical resection. Lung Cancer 2006;52:359-64.
17. Whitson BA, Groth SS, Duval SJ, et al. Surgery for early-stage non-small cell lung cancer: a systematic review of the video-assisted thoracoscopic surgery versus thoracotomy approaches to lobectomy. Ann Thorac Surg. 2008;86:2008-16.

18. Muroako M, Oka T, Tagawa T, et al. Video-assited thoracic surgery lobectomy reduces the morbidity after surgery for stage I non-small cell lung cancer. J Thorac Cardiovasc Surg. 2006;54:49-55.

19. Spicer JD, Shewale JB, Antonoff MB, et al. The influence of reconstructive technique on perioperative pulmonaryand infectious outcomes following chest wall resection. Ann Thorac Surg. 2016;102:1653-9.

20. Demmy TL, Nwogu CE, Yendamuri S. Thoracoscopic chest wall resection: what is its role? Ann Thorac Surg. 2010;89:S2142-5.

21. Drevet G, Ugalde PA. Uniportal video-assisted thoracoscopic surgery: safety, efficacy and learning curve during the first 250 cases in Quebec, Canada. Ann Cardiothorac Surg. 2016;5:100-6.

22. Cerfolio RJ, Bryant AS, Maniscalco LM. A nondivided intercostal muscle flap further reduces pain of thoracotomy: a prospective randomized trial. Ann Thorac Surg. 2008;85:1901-6.

23. Lee CY, Byun CS, Lee JG, et al. The prognostic factors of resected non-small cell lung cancer with chest wall invasion. World J Surg Oncol. 2012;10:9.

24. Strauss GM, Herndon JE II, Maddaus MA, et al. Adjuvant paclitaxel plus carboplatin compared with observation in stage IB non-small-cell lung cancer: CALGB 9633 with the Cancer and Leukemia Group B, Radiation Therapy Oncology Group, and North Central Cancer Treatment Group study groups. J Clin Oncol. 2008;26:5043-51.

25. Ettinger DS, Wood DE, Akerley W, et al. Non-small cell lung cancer, version 6. 2015. J Natl Compr Canc Netw. 2015;13:515-24.

26. Kawaguchi K, Yokoi K, Niwa $H$, et al. A prospective, multi-institutional phase II study of inductionchemoradiotherapy followed by surgery in patients with non-small cell lung cancer involving the chest wall (CJLSG0801). Lung Cancer 2017;104:7984. 